M.V. Fedoseenko ${ }^{1,2}$, D.A. Novikova ${ }^{1}$, N.E. Tkachenko ${ }^{1}$, M.I. Broeva ${ }^{1,}{ }^{2}$, T.A. Grechukha ${ }^{1}$, A.G. Gaivoronskaya ${ }^{1}$, V.V. Chernikov ${ }^{1}$

${ }^{1}$ Scientific Center of Children's Health, Moscow, Russian Federation

${ }^{2}$ Sechenov First Moscow State Medical University, Moscow, Russian Federation

\title{
Experience of application and safety assessment of the 13-valent pneumococcal conjugate vaccine in under- 5 children
}

\section{Author Affiliation:}

Marina Vladislavovna Fedoseenko, MD, senior research fellow at the vaccinal prevention department for children with medical issues at the research institute of preventive pediatrics and medical rehabilitation of the Scientific Center of Children's Health (Federal State Budgetary Research Institution)

Address: 2/62 Lomonosovskiy Av., Moscow, 119991; tel.: +7 (499) 134-20-92; e-mail: titovamarina@mail.ru

\section{Article received: 09.08.2014. Accepted for publication: 17.09.2014.}

Compulsory use of the 7-valent pneumococcal conjugate vaccine in the framework of national pediatric immunization schedules of the developed countries resulted in significant decrease in the prevalence of the pneumococcal infections induced by the vaccinal serotypes. However, a growth in prevalence of the pneumonia and acute otitis media caused by non-vaccinal strains has also been observed. This required introduction of a new 13-valent pneumococcal conjugate vaccine with a wider range of pneumococcal population coverage. The experience of application accumulated in various countries (2010 onwards) and results of the authors' observations indicate high safety of the 13-valent pneumococcal conjugate vaccine for both healthy under-5 children and patients with various medical issues. The article presents results of the 13-valent pneumococcal conjugate vaccination tolerance assessment. The study involved 110 children from 2 months to 5 years of age. In most cases immunization concurred with other pediatric vaccines. The incidence of local reactions in vaccinated children did not exceed 33\%, of generalized reactions $-11 \%$. The authors observed a comparable incidence of side reactions in both virtually healthy children and children with various medical issues.

Keywords: pneumococcal infection, vaccination, 13-valent pneumococcal conjugate vaccine (PCV13), vaccination safety, children.

\section{INTRODUCTION}

Outcomes of severe pneumococcal infection in children may be disastrous and, potentially, worse than outcomes of any other infections prevented with routine vaccinations. Moreover, mortality and incapacitation rates associated with severe pneumococcal infections remain higher than such rates associated with other vaccine-managed infections. Increasing resistance of the causative agent to antibacterial drugs complicates the matter further and emphasizes the need in a preventive approach. However, a visible downward pneumococcal infection mortality rate trend due to wide use of pneumococcal conjugate vaccines ought to be mentioned. According to the updated data of the World Health Organization as per 2008, up to $14.5 \mathrm{mn}$ cases of pneumococcal infection-induced diseases are detected in under-5 children annually [1]; on the average, 541,000 of them die. In 2000, mortality due to pneumococcal diseases was 1.5 times higher $-826,000$ [2]; it remains the highest in the developing countries of Africa and Asia, whereas in the developed countries of Europe and North America the mortality rate is tens of times lower.

The first pneumococcal conjugate vaccine with 7 most epidemiologically significant pneumococcal serotypes has been used for more than 10 years. Significant decrease in the incidence of invasive pneumococcal infections (IPIs) is associated with introduction of a 7- 
valent pneumococcal conjugate vaccine (PCV7) to schedules of compulsory pediatric vaccinations in the developed countries in the beginning of the 2000s. Decrease in the morbidity rate has been confirmed not only in the group of vaccinated infants, but also among adults due to the community immunity effect. Such a decrease in morbidity has been registered in the USA [3, 4], Canada [5], Australia [6], Germany [7], Norway [8]. The most important result of cohort PCV7 vaccination is lower incidence of severe pneumococcal infections - meningites and invasive infections in whole. E.g., in the USA - one of the first countries to introduce PCV7 to the national immunization schedule - the level of invasive pneumococcal diseases induced by the vaccinal serotypes decreased by $75 \%$ among the vaccinated infants and by $45 \%$ - in the unvaccinated population [3, 4]. From $1998 / 1999$ to $2004 / 2005$, the total rate of pneumococcal meningites in the USA decreased from 1.13 to 0.79 cases per 100,000 people; incidence of meningites of any etiology among under-2 children decreased by $64 \%$ (from 10.16 to 3.66 cases per 100,000 people) [9]. Naturally, the decrease in morbidity was primarily associated with the infections induced by the PCV7 pneumococcal serotypes (vaccinal serotypes). Other significant results of cohort PCV7 vaccination are a considerable decrease in occurrence of antibioticresistant pneumococcal strains and sanitation of pneumococcus carriers.

However, pneumococcus remains one of the primary causes of morbidity and mortality due to vaccination-preventable infections among under-5 children. It appeared that since spread of cohort vaccination the predominant pneumococci inducing severe invasive diseases, pneumonia and acute otitis media, have been non-vaccinal causative agent's strains, primarily 1, 3, 5, 6A and 19A. Analysis of 750 cases of invasive pneumococcal infections observed in PCV7vaccinated children demonstrated that the vaccination was ineffective only in $4 \%$ of the cases [3]. In all other cases IPIs were induced by non-vaccinal pneumococcal strains. In the USA the incidence of diseases induced by strain 19A increased from 0.8 to 2.5 cases per 100,000 people in 1998-2005. Thus, serotype 19A was the cause of 36\% of IPIs in under-5 children in 2005. Pneumococcal serotypes 1, 3 and 19A were the most commonly observed serotypes in American children with pneumonia; serotypes 19A and 6A caused the severest and the most protracted forms of acute otitis media. Moreover, pneumococcus 19A was the most antibiotic-resistant serotype.

Results of other clinical studies performed in 2005 demonstrated a vaccinal strain replacement phenomenon: decrease in prevalence and transmission of pneumococcal vaccinal serotypes in the presence of cohort PCV7 vaccination was accompanied by increase in the non-vaccinal serotype-induced pneumococcal diseases (pic. 1) [10].

Pic. 1. Rate of invasive pneumococcal diseases in under-5 children (USA, 1998-2004)

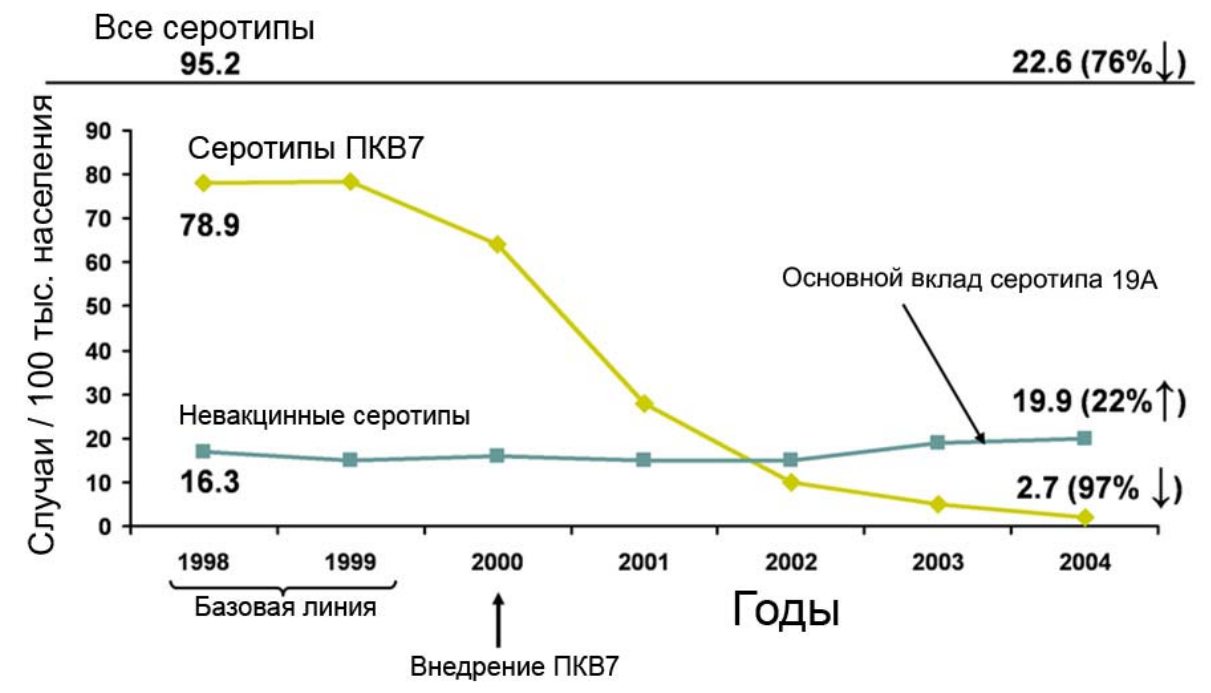


Note to the picture 1

$\begin{array}{ll}\text { Все серотипь } & \text { All serotypes } \\ \text { Случаи / } 100 \text { тыс. населения } & \text { Cases / 100,000 people } \\ \text { Годыл } & \text { Years } \\ \text { Базовая линия } & \text { Base line } \\ \text { Внедрение ПКВ7 } & \text { Introduction of PCV7 } \\ \text { Серотипь ПКВ7 } & \text { PCV7 serotypes } \\ \text { Невакционные серотипь } & \text { Non-vaccinal serotypes } \\ \text { Основной вклад серотипа 19A } & \text { Main contribution of serotype 19A }\end{array}$

The obtained results impelled researchers to create a pneumococcal conjugate vaccine with an extended spectrum of causative agent strains.

The 13-valent pneumococcal conjugate vaccine (PCV13) for preventive invasive pneumococcal diseases and acute otitis media covering the most common pneumococcal serotypes was licensed in February 2010 [11]. Along with the PCV7 serotypes (4, 6B, 9V, 14, 18C, 19F, 23F), PCV13 is also comprised of polysaccharides of serotypes 1,3, 5, 6A, 7F and 19A. Coverage of serotype $19 \mathrm{~A}$ by the 13-valent vaccine appears especially important due to its increasing role as a causative agent of invasive pneumococcal infections and increased antibiotic-resistibility.

According to the completed epidemiological studies, $71 \%$ of the circulating pneumococci around the world correspond to composition of the 7-valent pneumococcal vaccine, $84 \%$ of the serotypes - to the 10 -valent vaccine, $89 \%$ - to the 13 -valent vaccine [12]. The data correspond with results of the studies performed in 8 Federal Districts of the Russian Federation, i.e. the correspondence of the pneumococcal serotypes detected in carriers with the pneumococcal conjugate vaccinal serotypes: $80.4,80.7$ and $84.6 \%$ for $7-, 10$ - and 13-valent vaccines, respectively [13]. According to a large-scale study (2010/2011) of infants in Moscow, the spectrum of pneumococcal serotypes observed in carriers corresponded with the PCV serotypes by $60.3,64.2$ and $94.5 \%$, respectively [12]. According to structural analysis of the serotypes detected in middle ear fluid in patients with otitis media, serotype spectrum correspondence was $65.8,67.4$ and $93.8 \%$ for $7-, 10$ - and 13 -valent vaccines, respectively.

Immunogenicity and clinical effectiveness of the pneumococcal conjugate vaccine have been proved with large-scale clinical studies performed in different countries; most importantly, they have been confirmed with world clinical practice [9].

Moreover, PCV13 antigen spectrum extension did not affect the modified vaccine's safety profile, according to results of the clinical studies performed both in the course of vaccine licensing [11] and its further field use.

Safety of the 13-valent conjugate vaccine has been confirmed with results of a meta-analysis based on 13 clinical studies performed in 9 countries of North America, Europe and Asia [14, 15]. The study involved more than 4,720 healthy children, who received at least 1 PCV13 vaccine dose together with other national calendar vaccines, and 2,760 infants vaccinated with PCV7. Local and general reactions were assessed 4-7 days after administration of each vaccinal dose and registered in electronic cards. The most reportable ( $>20 \%$ of the cases) reactions within the first 7 post-vaccinal days were pain, reddening and densification of the injection site, fever, anorexia, irritability and sleep disorder. Along with that, the PCV13 safety data were compared to the 7-valent conjugate vaccine safety data. In general, the frequency of local reactions after any of the primary PCV13 doses or PCV7 was comparable: painful injection site (46.7 and 44.7\%, respectively), lump (28.5 and 26.9\%) and reddening (36.4 and $33.9 \%$ ). On the other hand, painfulness was observed significantly more often among the PCV-7 vaccinated children after revaccination in the second year of life $(54.4$ and $48.8 \% ; p=0.005)$. Fever $(38-39$ $\left.{ }^{\circ} \mathrm{C}\right)$ rate in the post-vaccinal period was comparable (16.5-28\% on the average) and characterized by moderate course and rapid reversal.

Another safety assessment study involved 354 7-71-months-old infants who received at least 1 PCV13 dose together with other pediatric vaccines [9]. The control group consisted of 
284 15-59-months-old healthy children who have previously received 1 dose or undergone the complete PCV7 schedule. Comparative observation of PCV7- and PCV13-vaccinated children indicated a comparable amount of adverse post-immunization reactions.

Comparative tolerance analysis of PCV13 and PCV7 performed in Kentucky (USA) involved 250 infants, confirmed a comparable rate of adverse reactions induced by both vaccines and demonstrated a range of peculiarities. Thus, the rate of local and several general reactions (anorexia, sleep disorder, anxiety) was the highest after the first vaccinal dose and reduced after each subsequent administration. Marked painfulness in the injection site was the most reportable complication after the second vaccinal dose in the PCV7 group (not typical of the PCV13 group) [16].

A comparative study of two pneumococcal conjugate vaccines (Italy) utilized a $2+1$ schedule and, primarily, combined vaccination [17]. These vaccination rules are similar to the real National calendar practice in the Russian Federation. In general, comparative tolerance characteristics of two conjugate vaccines were similar and proved high safety level of PCV13. Significant differences were observed only regarding the anxiety rate after the first dose, which was higher in the PCV13 group (72.9 and 63.7\% [PCV7 group], respectively). Moderate fever was observed significantly more often in the PCV13-revaccinated infants (63.7 and 52.3\%).

Such clinical studies of PCV13 reactogenicity in comparison with PCV7 tolerance parameters have been performed in many countries [18-21]. The results confirm high safety level of PCV13, which is as high as of precedent PCV7. Immunization was almost always characterized by simultaneous administration of other pediatric vaccines; this confirms the absence of effect of additional PCV13 prescription on tolerance to the primary calendar vaccines. A clinical study performed in Brazil, where PCV13 was administered simultaneously with a whole cell DTP vaccine, is of high interest [22]. The obtained results demonstrated a reliable immune response to all the administered antigens and safety of use of PCV13 together with the main pediatric vaccine.

Despite the fact that the risk of post-vaccinal reactions was determined in the course of a pre-licensing clinical study of the PCV13 vaccine, monitoring of rare severe adverse postvaccinal complications is going on in the USA. The PCV13 safety monitoring system was launched immediately after the vaccine had been introduced to the national immunization calendar. It has been confirmed that PCV13 vaccination does not result in high risk of febrile convulsions, urticaria, angioneurotic edema, anaphylactic shock, asthma and thrombocytopenia [23].

In Russia, the PCV13 vaccine was licensed in the end of 2011 and has completely replaced the precedent PCV7 vaccine. Data on the use of pneumococcal conjugate vaccines for several regions of Russia have already been made public; more than ten analytical reports on PCV7/13 immunization safety have been published on the basis of clinical observations.

In particular, the review by S.V. Ilyina et al. [24] cites an analysis of publications dedicated to the experience of use of pneumococcal conjugate vaccines in infants in different regions of the Russian Federation. These data confirmed effectiveness and high tolerance to PCV7/13 in the event of the administration thereof simultaneously with other Russian national preventive vaccination calendar vaccines. Most studies involved risk group children: children from infant orphanage and needy families, frequently ill children, premature infants, children with congenital heart diseases, bronchopulmonary dysplasia and HIV.

\section{PATIENTS AND METHODS}

A study of PCV13 vaccination tolerance among under-5 children was performed in 2013/2014 at the vaccinal prevention unit for children with health deviations at the Scientific Center of Children's Health. 110 children were vaccinated in the course of the study; 146 PCV13 vaccine doses were used.

\section{Study methods}


PCV13 vaccine was administered intramuscularly to the anterolateral thigh surface (infants under 24 months of age) or deltoid muscle (over-2 children) in the dose of $0.5 \mathrm{ml}$. Children would receive one, two, three or four PCV13 doses depending on the age at beginning of vaccination. PCV13 vaccination was performed according to the standard schedule (see instruction for use) $-3+1$ for 2-6-months-old infants and $2+1$ for 7-11-months-old children. 12-23-months-old children were vaccinated twice (with the minimal interval of 2 months); over2 children received 1 vaccinal dose. The vaccinated children were directly observed at the unit for 30 post-vaccinal minutes; after that, control of general and local reactions was performed by means of a phone call on the $4^{\text {th }}$ post-vaccinal day. Drug therapy in the post-vaccinal period was prescribed when necessary depending on the initial medical condition.

\section{Statistical data manipulation}

Statistical data were manipulated using non-parametric criteria and calculating the MannWhitney U test.

\section{RESULTS}

The average age of the vaccinated children at beginning of vaccination was 20 months. The youngest vaccinated child was 2 months of age, the oldest -5.5 years. Distribution of children by age at beginning of vaccination is given in pic. 2 .

Pic. 2. Age at beginning of PCV13 vaccination

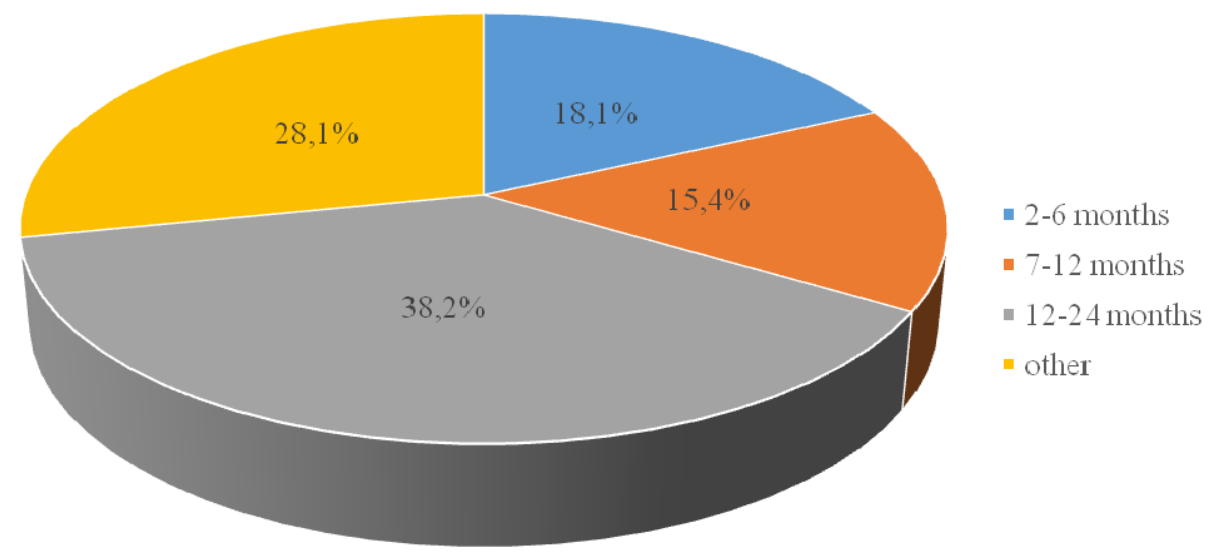

Late beginning of vaccination, primarily in over-1 children, is, apparently, due to a high share of patients with concurrent diseases, which is why these children undergo a prolonged vaccination exemption (until condition) stabilization. Moreover, due to the parents' cautious attitude to vaccination, most of them consent to introduction of an additionally prescribed vaccine only after the primary vaccination schedule has been completed - DTP + poliomyelitis + hepatitis B. Interestingly, the share of children vaccinated with other vaccines increases with each PCV13 dose (pic. 3). This may indicate increase in trust in the PCV13 vaccine among parents observed in the process of immunization.

It ought to be mentioned, however, that, according to the WHO recommendations, the maximal protective effect of pneumococcal vaccination is achieved when such a vaccination is performed to all under-2 children and as early as possible (due to high risk of pneumococcal infections in infants from birth) [1]. Moreover, numerous studies of children with different forms of concurrent diseases confirmed high level of PCV13 effectiveness and safety [24, 25]. 
Pic. 3. Combined vaccination percentage depending on the PCV13 dose

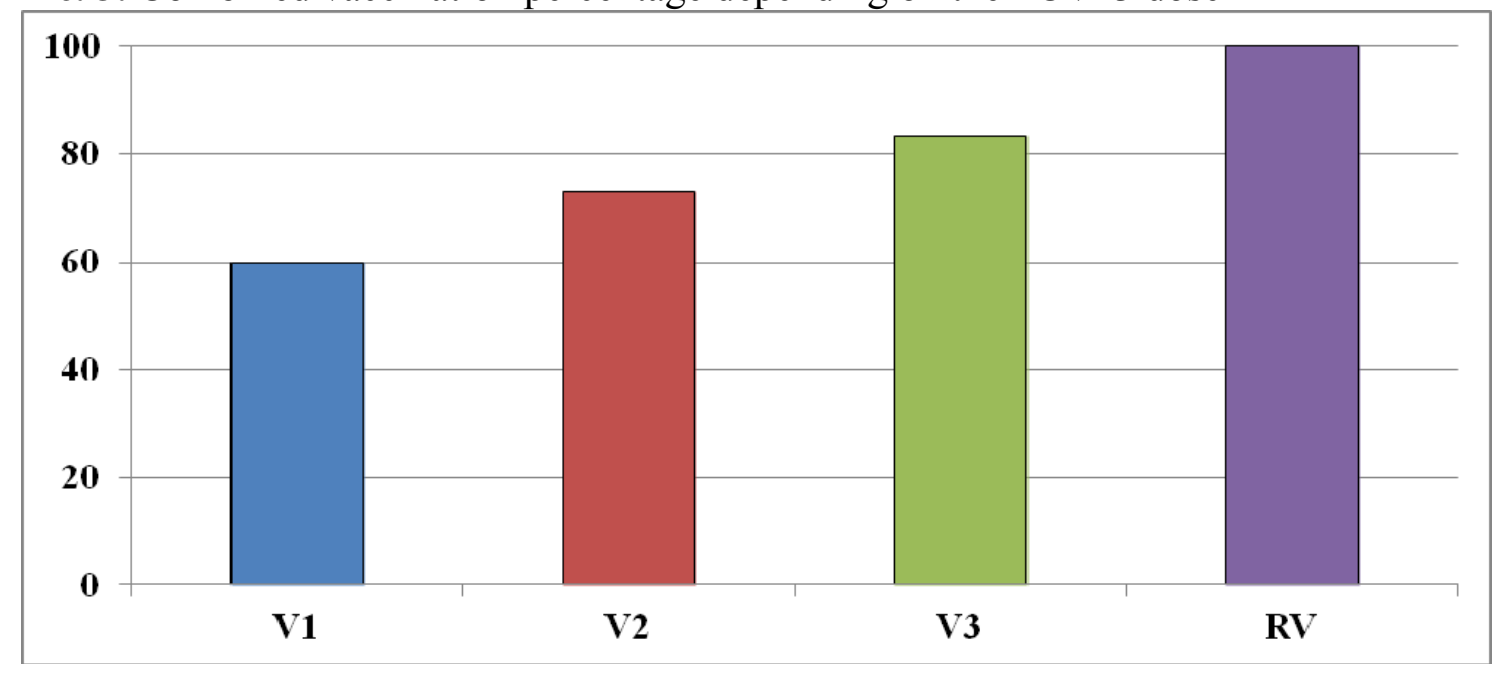

A significant number of the vaccinated patients in this study $(60.4 \%)$ had various pathologies, the most common of them being allergic forms of diseases, pathologies of ENT organs, cardiovascular (congenital heart disease) and bronchopulmonary systems (bronchial asthma, bronchopulmonary dysplasia), consequences of perinatal lesion of the central nervous system and hereditary diseases (hereditary spherocytosis, cleft soft palate, celiac disease).

In most cases (64\%), immunization was performed simultaneously with other pediatric vaccines. The most common concurrent vaccine was a combined DTaP-IPV/Hib vaccine. The following vaccines were administered simultaneously with PCV13 more rarely (in descending order): OPV, rotavirus vaccine and a three-valent vaccine for measles, parotitis and rubella.

The post-vaccinal period was uncomplicated in most children $(71 ; 64.5 \%)$. The most common adverse events in the post-vaccinal period were local reactions in the form of reddening and densification of the vaccine injection sit (tb. 1). On the average, one out of three vaccinations $(32.9 \%)$ was characterized by development of local reactions; this corresponds to the world clinical practice data $[4,11,16,17]$. Almost all reactions were mild or moderate and subsided within 3 days in the setting of appropriate palliative care ad did not require medical care. Intense local reactions in the form of densification and hyperemia larger than $5 \mathrm{~cm}$ in diameter were observed only in 3 children (2.7\%). General reactions (body temperature rise, clinical symptoms of general malaise) were observed in $10.9 \%$ of the cases (tb. 2); fever was observed especially often in the children vaccinated with the first PCV13 dose, whereas in the post-vaccinal period (after revaccination and the third vaccination) the body temperature would not rise. Severity of general reactions in all cases was mild or moderate. Body temperature did not exceed $38.5{ }^{\circ} \mathrm{C}$ and could be alleviated with single dose of an antifebrile drug.

Table 1. Local reactions in the PCV13 post-vaccinal period depending on the amount of administered vaccinal doses

\begin{tabular}{|c|c|c|c|c|c|}
\hline \multirow{2}{*}{ Dose } & Other & Reddening & Densification & Painfulness & Edema \\
\cline { 2 - 6 } & \multicolumn{5}{|c|}{ n (\%) } \\
\hline V1 & $39(35.4)$ & $29(26.3)$ & $10(9.1)$ & $1(0.9)$ & $5(4.5)$ \\
\hline V2 & $7(26.9)$ & $3(11.5)$ & $6(23.1)$ & $1(3.8)$ & $2(7.7)$ \\
\hline V3 & $1(16.7)$ & $1(16.7)$ & $1(16.7)$ & - & - \\
\hline RV & $1(20)$ & - & $1(20)$ & - & - \\
\hline
\end{tabular}

Note. $\mathrm{n}$ - number of children with a specific reaction. 
Table 2. General reactions to the PCV13 vaccination depending on the amount of administered doses

\begin{tabular}{|c|c|c|c|c|c|}
\hline \multirow{2}{*}{ Dose } & Other & Fever & Capriciousness & Atony & Sleep disorder \\
\cline { 2 - 6 } & \multicolumn{5}{|c|}{ n (\%) } \\
\hline V1 & $13(11.8)$ & $13(11.8)$ & $4(3.6)$ & $4(3.6)$ & $3(2.7)$ \\
\hline V2 & $3(11.5)$ & $3(11.5)$ & $1(3.8)$ & - & - \\
\hline V3 & - & - & - & - & - \\
\hline RV & - & - & - & - & - \\
\hline
\end{tabular}

Note. $\mathrm{n}$ - number of children with a specific reaction.

No increase in the rate of post-vaccinal reactions was observed after each subsequent administration. On the contrary, the rate of local reactions decreased significantly (see tbs. 1,2) after the second PCV13 dose onwards; general medical condition did not aggravate in the post-vaccinal period after administration of the last PCV13 doses (V3 and RV) in any of the patients.

Immunization tolerance was compared between healthy children and children with various pathologies. Adverse reactions after PCV13 vaccination were observed in $36.8 \%$ of the cases in healthy children and in $44.4 \%$ of the cases in patients with health deviations; however, no significant differences were observed. Neither child suffered exacerbation of the primary disease in the post-vaccinal period.

Thus, moderate local reactions and short-term body temperature rise up to low-grade fever were detected in more than half of the frequently ill children and the patients under otorhinolaryngological observation $(62.5 \%)$, as identified by a previous study of tolerance of the 7 -valent pneumococcal conjugate and the 23 -valent polysaccharidic vaccines performed at our unit [26]. Apparently, high risk of local reactions in the persons who have previously had pneumococcal infections may be caused by an initially high concentration of specific antibodies. On the contrary, in children with hereditary diseases, congenital heart disease and a patient with Marshall syndrome and celiac disease vaccination was asymptomatic in all cases.

\section{CONCLUSION}

PCV13 vaccine represents the newest generation of pneumococcal conjugate vaccines with the broadest coverage spectrum. In numerous clinical studies of healthy infants performed in different countries PCV13 was characterized by a favorable safety profile (comparable with safety profile of the precedent vaccine, PCV7).

We have also been able to confirm safety of the use thereof. Results of a performed study of under-5 children demonstrated safety of a 13-valent pneumococcal conjugate vaccine. It also demonstrated that PCV13 is well tolerated both by healthy children and patients with different pathologies.

\section{FINANCIAL DISCLOSURE}

The study was financially supported by Pfizer.

\section{REFERENCES}

1. Estimated Hib and pneumococcal deaths for children under 5 years of age, 2008. World Health Organization. 2012; March. Available at: http://apps.who.int/immunization_monitoring/burden/Pneumo_hib_estimates/en/index.html 
2. WHO Global Immunization Vision and Strategy, April 2005. Available at: www.who.int/vaccines/GIVS/english/Global_imm._data_EN.pdf

3. Bocchini J.A. Jr., Bradley J.S., Brady M.T., Bernstein H.H., Byington C.L., Fisher M.C., Glode M.P., Jackson M.A., Keyserling H.L., Kimberlin D.W., Orenstein W.A., Schutze G.E., Willoughby R.E. Jr. Recommendations for the Prevention of Streptococcus pneumonia Infections in Infants and Children: Use of 13-valent Pneumococcal Conjugate Vaccine and Pneumococcal Polysaccaride Vaccine. Pediatrics. 2010 Jul; 126 (1): 186-90.

4. Paradiso P.R. Advances in pneumococcal disease prevention: 13-valent Pneumococcal Conjugate Vaccine for Infants and Children. Clin Infect Dis. 2011; 52 (10): 1241-1247.

5. Bettinger J.A., Scheifele D.W., Kellner J.D., Halperin S.A., Vaudry W., Law B., Tyrrell G., Morris R., Halperin S., Dery P., Moore D., Lebel M., Le Saux N., Tran D., Embree J., Tan B., Jadavji T., Vaudry W., Sauve L. The effect of routine vaccination on invasive pneumococcal infections in Canadian children, Immunization Monitoring Program, Active 2000-2007. Vaccine. 2010; 28: 2130-6.

6. Lehmann D., Willis J., Moore H.C., Moore H.C., Giele C., Murphy D., Keil A.D., Harrison C., Bayley K., Watson M., Richmond P. The changing epidemiology of invasive pneumococcal disease in aboriginal and non-aboriginal western Australians from 1997 through 2007 and emergence of nonvaccine serotypes. Clin Infect Dis. 2010; 50: 1477-86.

7. Rückinger S., van der Linden M., Reinert R.R., von Kries R., Burckhardt F., Siedler A. Reduction in the incidence of invasive pneumococcal disease after general vaccination with 7valent pneumococcal conjugate vaccine in Germany. Vaccine. 2009; 27: 4136-41.

8. Vestrheim D.F., Lovoll O., Aaberge I.S., Caugant D.A., Hoiby E.A., Bakke H., Bergsaker M.R. Effectiveness of a $2+1$ dose schedule pneumococcal conjugate vaccination programme on invasive pneumococcal disease among children in Norway. Vaccine. 2008; 26: $3277-81$.

9. Food and Drug Administration. Vaccines: approved products. Prevenar 13 (pneumococcal 13-valent conjugate vaccine). Available at: http://www.fda.gov/BiologicsBloodVaccines/Vaccines/ApprovedProducts/ucm201667.htm. Accessed March 5, 2010.

10. Fedoseenko M.V., Namazova-Baranova L.S. International experience of pneumococcal conjugate vaccines: challenges, achievements and prospects. Voprosy sovremennoi pediatrii $=$ Current Pediatrics. 2009; 8 (1): 136-9.

11. Licensure of a 13-Valent Pneumococcal Conjugate Vaccine (PCV13) and Recommendations for Use Among Children - Advisory Committee on Immunization Practices 
(ACIP), 2010. Morbidity and Mortality Weekly Report (MMWR). Weekly. 2010 March 12; 59 (09): $258-61$.

12. Baranov A.A., Namazova-Baranova L.S., Mayanskii N.A. et al. The role of Streptococcus pneumoniae in the structure of bacterial infections in children admitted to hospitals in Moscow in 2011-2012. Pediatricheskaya farmakologiya = Pediatric pharmacology. 2013; 10 (5): 6-13.

13. Kozlov R.S. Pnevmokokkovaya infektsiya: sovremennye podkhody k profilaktike i terapii. Lektsii dlya praktikuyushchikh vrachei [Pneumococcal Disease: Current Approaches to Prevention and Therapy. Lectures for Practitioners]. Moscow, Zdorov'e cheloveka, 2005. P. 219-234.

14. Thompson A., Gurtman A., Patterson S. Safety of 13-valent pneumococcal conjugate vaccine in infants and children: meta-analysis of 13 clinical trials in 9 countries. Vaccine. 2013 Oct 25; 31 (45): 5289-95.

15. Ruiz-Aragón J. Safety and immunogenicity of 13-valent pneumococcal conjugate vaccine in infants: A meta-analysis. Vaccine. 2013; 31: 5349-58.

16. Bryant K.A., Block S.L., Baker S.A. Safety and immunogenicity of a 13-valent pneumococcal conjugate vaccine. Pediatrics. 2010 May; 125 (5): 866-75.

17. Esposito S., Tansey S., Thompson A. Safety and Immunogenicity of a 13-Valent Pneumococcal Conjugate Vaccine Compared to Those of a 7-Valent Pneumococcal Conjugate Vaccine Given as a Three-Dose Series with Routine Vaccines in Healthy Infants and Toddlers. Clin Vaccine Immunol. 2010 Jun; 17 (6): 1017-26.

18. Vanderkoii O.G., Scheifele D.W., Girgenti D., Halperin S.A., Patterson S.D., Gruber W.C., Emini E.A., Scott D.A., Kellner J.D. Safety and immunogenicity of 13-valent pneumococcal conjugate vaccine in healthy infants and toddlers given with routine pediatric vaccination in Canada. Pediatr Infect Dis J. 2012 Jan; 31 (1): 72-7.

19. Kieninger D.M., Kueper K., Steul K., Juergens C., Ahlers N., Baker S., Jansen K.U., Devlin C., Gruber W.C., Emini E.A., Scott D.A. Safety, tolerability and immunologic noninferiority of a 13-valent pneumococcal conjugate vaccine compared to a 7-valent pneumococcal conjugate vaccine given with routine pediatric vaccination in Germany. Vaccine. 2010 Jun; 7 (28): 25.

20. Togashi T., Yamaji M., Thompson A., Giardina P.C., Aizawa M., Patterson S., Gruber W.C., Scott D.A. Immunogenicity and safety of 13-valent pneumococcal conjugate vaccine in health infants in Japan. Pediatr Infect Dis J. 2013 Sep; 32 (9): 984-9. 
21. Gutierez Brito M., Thompson A., Girgenti D., Giardina P.C., Sarkozy D.A., Gruber W.C., Emini E.A., Scott D.A. Immunogenicity and safety of 13-valent pneumococcal conjugate vaccine in Mexico. Rev Panam Salud Publica. 2013 Jun; 33 (6): 414-21.

22. Weckx L.Y., Thompson A., Berezin E.N., de Faria S.M., da Cunha C.A., Pride M., Patterson S., Gruber W.C., Emini E.A., Scott D.A. A phase 3, randomized, double-blind trial comparing the safety and immunogenicity of the 7-valent and 13-valent pneumococcal conjugate vaccines, given with routine pediatric vaccinations, in healthy infants in Brazil. Vaccine. 2012 Dec 14; 30 (52): 7566-72.

23. Tseng H.F., Sy L.S., Liu I.L., Qian L., Marcy S.M., Weintraub E., Yih K., Baxter R., Glanz J.M., Donahue J., Naleway A., Nordin J., Jacobsen S.J. Postlicenzure surveillance for prespecified adverse events following the 13-vallent pneumococcal conjugate vaccine in children. Vaccine. 2013 May 24; 31 (22): 2578-83.

24. Il'ina S.V., Beletskaya O.A., Sabitov A.U., Chernaya N.L., Snegova N.F., Salkina O.A., Dzhumagaziev A.A., Bakhareva N.V., Brovkina A.A., Savvina N.V. The results of evaluation of the effectiveness and safety of conjugate pneumococcal vaccines in the Russian Federation. Epidemiologiya $i$ infektsionnye bolezni, aktual'nye voprosy = Epidemiology and infectious diseases, topical issues. 2013; 6: 55-60.

25. Il'ina S.V., Lysanov Yu.I. Vaccination Pneumococcal conjugate vaccine in preterm infants and children with congenital heart disease in Irkutsk. Pediatricheskaya farmakologiya = Pediatric pharmacology. 2013; 10 (3): 12-16.

26. Gaivoronskaya A.G., Namazova-Baranova L.S., Galitskaya M.G., Grechukha T.A., Tkachenko N.E., Ivardava M.I. Analysis of the efficacy and safety of vaccination against pneumococcal disease for children with various disabilities in health. Pediatricheskaya farmakologiya $=$ Pediatric pharmacology. 2012; 9 (2): 10-14. 